\title{
A CONTINUITY THEOREM FOR FUCHSIAN GROUPS $\left(^{1}\right)$
}

\author{
BY \\ C. K. WONG
}

\begin{abstract}
On a given Riemann surface, fix a discrete (finite or infinite) sequence of points $\left\{\boldsymbol{P}_{k}\right\}, k=1,2,3, \ldots$, and associate to each $\boldsymbol{P}_{k}$ an "integer" $\nu_{k}$ (which may be $1,2,3, \ldots$, or $\infty$ ). This sequence of points and "integers" is called a "signature" on the Riemann surface. With only a few exceptions, a Riemann surface with signature can always be represented by a Fuchsian group. We investigate here the dependence of the group on the number $\nu_{k}$. More precisely, keeping the points $\boldsymbol{P}_{k}$ fixed, we vary the numbers $v_{k}$ in such a way that the signature tends to a limit signature. We shall prove that the corresponding representing Fuchsian group converges to the Fuchsian group which corresponds to the limit signature.
\end{abstract}

I. Introduction. On a given Riemann surface, fix a discrete (finite or infinite) sequence of points $\left\{\boldsymbol{P}_{k}\right\}, k=1,2,3, \ldots$, and associate to each $\boldsymbol{P}_{k}$ an "integer" $\nu_{k}$ (which may be $1,2,3, \ldots$, or $\infty$ ). This sequence of points and "integers" is called a "signature" on the Riemann surface. With only a few exceptions, a Riemann surface with signature can always be represented by a Fuchsian group. This is the celebrated limit circle theorem of Koebe. We investigate here the dependence of the group on the numbers $v_{k}$. More precisely, keeping the points $P_{k}$ fixed we vary the numbers $\nu_{k}$ in such a way that the signature tends to a limit signature. We shall prove that the corresponding representing Fuchsian group converges to the Fuchsian group which corresponds to the limit signature, in a sense which we shall specify in the text.

This main result is established in §VII.

II. Preliminaries. In the first part of this section we state the limit circle theorem of Koebe [10]. The rest of this section is devoted to the statement and proof of some properties of the solution of an elliptic partial differential equation.

Definition 1. Let $S$ be a Riemann surface and $\left\{P_{k}\right\}, k=1,2,3, \ldots$, be a discrete (finite or infinite) sequence of points on $S$. Let there be an "integer" $v_{k} \geqq 2$ associated with each point $P_{k}$ (here $\nu_{k}$ may be an actual integer, or the symbol $\infty$ ). The following two cases are excluded: (i) $S=\hat{C}=C \cup\{\infty\}$, the Riemann sphere, with one point $P$ and the associated "integer" $\nu \neq \infty$ and (ii) $S=\hat{C}$, with two points $P_{1}, P_{2}$

Received by the editors July 24, 1970 and, in revised form, May 18, 1971.

AMS 1969 subject classifications. Primary 3045, 3030.

Key words and phrases. Riemann surfaces with signature, the limit circle theorem of Koebe, Fuchsian groups, Poincaré metrics.

( ${ }^{1}$ This paper is a modified version of the author's doctoral dissertation at Columbia University, 1970. The author is much indebted to Professor Lipman Bers for his guidance.

Copyright (C) 1972, American Mathematical Society 
and $\nu_{1} \neq \nu_{2}$. The triple $\left(S,\left\{P_{k}\right\},\left\{\nu_{k}\right\}\right)$ is called a Riemann surface with signature. For simplicity, we shall write $\boldsymbol{P}, \boldsymbol{v},(S, \boldsymbol{P}, \boldsymbol{v})$ for $\left\{\boldsymbol{P}_{k}\right\},\left\{\nu_{k}\right\},\left(S,\left\{\boldsymbol{P}_{k}\right\},\left\{\nu_{k}\right\}\right)$ respectively.

TheOREM 1 (KoEBe). Let $(S, \boldsymbol{P}, \nu)$ be a Riemann surface with signature. Let $S^{\#}=S-\bigcup_{v_{k}=\infty}\left\{P_{k}\right\}, \quad S^{b}=S-\bigcup_{v_{k} \geqq 2}\left\{P_{k}\right\}$.

Then there exists a simply connected Riemann surface $V^{\#}$, a properly discontinuous group $G$ of conformal selfmappings of $V^{\#,}$ and a conformal mapping from $V \# / G$ onto $S \#$ such that

(i) under this mapping $S^{b}$ is conformally equivalent to $V^{b} / G$, where $V^{b}$ is $V^{\#}$ with all fixed points of elements of $G$ removed, and

(ii) the natural projection $V^{\#} \rightarrow V^{\#} / G$ followed by the conformal mapping $V^{\# / G ~}$ $\rightarrow S^{\#}$ is locally 1-1 at each point of $V^{b}$ and is $\nu_{k}$ to 1 at the preimages of $P_{k}$ with $\nu_{k}<\infty$.

The group $G$ is determined uniquely up to conjugation in the full group of conformal selfmappings of $V^{\#} .\left({ }^{2}\right)$

REMARK. The fact that $V^{\#}$ is simply connected implies that $V^{\#}$ is conformally equivalent to $\hat{C}, \boldsymbol{C}$ or $\Delta$, where $\Delta$ denotes, here and hereafter, the unit disk. We can actually enumerate all Riemann surfaces with signature $(S, P, v)$ such that $V^{\#}$ is conformally equivalent to $\hat{C}$ or $C$ as follows. (See Ford [7, Chapter IX, \$94, 95].)

(i) $S=\hat{C}$ with one point $P, \nu=\infty$,

(ii) $S=\hat{C}$ with two points $P_{1}, P_{2}$, and $\nu_{1}=\nu_{2}$,

(iii) $S=\hat{C}$ with three points $P_{1}, P_{2}, P_{3}$, and $1 / \nu_{1}+1 / \nu_{2}+1 / \nu_{3} \geqq 1$,

(iv) $S=\hat{C}$ with four points $P_{1}, P_{2}, P_{3}, P_{4}$ and $\nu_{1}=\nu_{2}=\nu_{3}=\nu_{4}=2$,

(v) $S=$ a torus and there are no points $P_{k}$.

We shall call these cases exceptional.

COROllary. Given a nonexceptional Riemann surface with signature $(S, P, \nu)$, there exists a Fuchsian group $G$ with the unit circle as its fixed circle such that $S \#=S-\bigcup_{v_{k}=\infty}\left\{P_{k}\right\}$ is conformally equivalent to $\Delta / G . S^{b}=S-\bigcup_{v_{k} \geqq_{2}}\left\{P_{k}\right\}$ is conformally equivalent to $\Delta_{G} / G$, where $\Delta_{G}=\Delta-\{$ all elliptic fixed points of $G\}$, and $S$ is conformally equivalent to $\hat{\Delta}_{G} / G$, where $\hat{\Delta}_{G}=\Delta \cup$ \{all parabolic fixed points of $G$ \}. The natural projection $\Delta \rightarrow \Delta / G$ followed by the conformal mapping $\Delta / G \rightarrow S \#$ is locally 1-1 at each point of $\Delta_{G}$, and is $\nu_{k}$ to 1 at the preimages of $P_{k}$ with $\nu_{k}<\infty$.

The Fuchsian group $G$ is determined uniquely up to conjugation by a Möbius transformation.

Proof. We have only to show that $S$ is conformally equivalent to $\hat{\Delta}_{G} / G$. But this is exactly a result of Heins [8].

In this case we say that $(S, P, v)$ is represented by a Fuchsian group $G$. We shall also allow $\nu_{k}=1$. That is, given a Riemann surface $S$ and a discrete (finite or

(2) A complete proof of the limit circle theorem of Koebe can be found in the abovementioned dissertation. 
infinite) sequence of points $\left\{P_{k}\right\}, k=1,2,3, \ldots$, on $S$, we associate an "integer" $\nu_{k}$ to each point $P_{k}$, where $\nu_{k} \geqq 1$ or $=\infty$.

Definition 2. (i) A triple $(S, \boldsymbol{P}, \boldsymbol{\nu})$ is called a Riemann surface with signature if after ignoring all points $P_{k}$ with $\nu_{k}=1$, the resulting triple is a Riemann surface with signature as in Definition 1.

(ii) A triple $(S, P, v)$ is called exceptional if after ignoring all points $P_{k}$ with $\nu_{k}=1$, the resulting triple is an exceptional Riemann surface with signature, as defined above.

We shall discuss only nonexceptional triples throughout this paper.

The rest of this section will be devoted to the study of some properties of realvalued solutions of the elliptic partial differential equation

$$
\left(\partial^{2} / \partial t \partial \bar{t}\right) \log \lambda(t)=\lambda^{2}(t)
$$

or, equivalently,

$$
\left(\partial^{2} / \partial t \partial \bar{t}\right) u(t)=e^{2 u(t)}
$$

where $\lambda(t), u(t)$ are real-valued functions of complex-variable $t$, and $\lambda=e^{u}$. From now on whenever we say that $u$ satisfies (2) we mean that $u$ is a real-valued $C^{2}$ solution of (2). The proof of Lemmas 1, 2, 3 and 4 can be accomplished by standard potential-theoretical arguments as in [2] and is therefore omitted.

LEMMA 1. If $u_{1}, u_{2}$ satisfy (2) in some domain $D$, then $u_{1}-u_{2}$ has neither positive maxima nor negative minima in $D$.

LEMMA 2. If $\left\{u_{i}\right\}, i=1,2,3, \ldots$, satisfy (2) in $|t|<R$, and for every $i,\left|u_{i}\right| \leqq M$ there, then in $|t|<R_{0}<R$, any infinite sequence of the family $\left\{u_{i}\right\}$ has a uniformly convergent subsequence.

LEMMA 3. If $\left\{u_{i}\right\}, i=1,2,3, \ldots$, satisfy (2) in $|t|<R$ and $u_{i} \rightarrow u$ uniformly in $|t|<R$ as $i \rightarrow \infty$, then $u$ is of class $C^{2}$ and satisfies (2) in $|t|<R_{0}<R$.

Corollary. If $\left\{u_{i}\right\}, i=1,2,3, \ldots$, satisfy (2) in $D$ and $u_{i} \rightarrow u$ normally in $D$ as $i \rightarrow \infty$, then $u$ is of class $C^{2}$ and satisfy (2) in $D$.

LEMMA 4. If $u$ satisfies (2) in $0<|t|<R$ and $u$ is bounded, then $u$ is of class $C^{2}$ in $|t|<R$ and satisfies (2) there.

LEMMA 5. If $u(t)$ satisfies (2) in $0<|t|<R$ and $u(t) \sim b+(1 / n-1) \log |t|$, as $t \rightarrow 0$, where $b$ is a constant and $n$ is a positive integer, then there exists a function $v(z),|z|<R^{1 / n}$, such that $v(z)$ is of class $C^{2}$, satisfying $(2), v\left(e^{2 \pi i / n} z\right)=v(z)$ for $|z|<R^{1 / n}$ and $v(z)=u\left(z^{n}\right)+\log n+(n-1) \log |z|$ for $0<|z|<R^{1 / n}$.

Proof. Set $z=t^{1 / n}$. Define $v(z)=u\left(z^{n}\right)+\log n+(n-1) \log |z|$ for $0<|z|<R^{1 / n}$. Then clearly $v(z)$ is well defined and $v\left(e^{2 \pi i / n} z\right)=v(z)$. By assumption, $u\left(z^{n}\right) \sim b$ $+(1-n) \log |z|$, as $z \rightarrow 0$. Hence $v(z) \sim b+\log n$ as $z \rightarrow 0$. Therefore $v(z)$ is bounded 
in $0<|z|<R^{1 / n}$. But $v(z)$ is of class $C^{2}$ in $0<|z|<R^{1 / n}$, and satisfies (2) there; by Lemma $4, v(z)$ is of class $C^{2}$ in $|z|<R^{1 / n}$ and satisfies (2) there.

Corollary. If $\lambda(t)$ is a positive $C^{2}$ solution of (1) in $0<|t|<R$ and $\lambda(t)$ $\sim a|t|^{1 / n-1}$ as $t \rightarrow 0$, where $a>0$ is a constant and $n$ is a positive integer, then there exists a function $\mu(z),|z|<R^{1 / n}$, such that $\mu(z)$ is positive, of class $C^{2}$, satisfying (1), $\mu\left(e^{2 \pi i / n} z\right)=\mu(z)$ for $|z|<R^{1 / n}$ and $\mu(z)=\lambda\left(z^{n}\right) n|z|^{n-1}$ for $0<|z|<R^{1 / n}$.

LEMMA 6. If $\left\{u_{i}\right\}, i=1,2,3, \ldots,\left\{\hat{u}_{j}\right\}, j=1,2$, satisfy (2) in $0<|t|<R, \hat{u}_{1} \leqq u_{i} \leqq \hat{u}_{2}$ in $0<|t|<R$ for all $i, u_{i}(t) \sim b_{i}+(1 / n-1) \log |t|, \hat{u}_{j}(t) \sim \hat{b}_{j}+(1 / n-1) \log |t|$, as $t \rightarrow 0$, where $b_{i}, \hat{b}_{j}$ are constants, $n$ is a positive integer, and finally, $u_{i} \rightarrow u$ normally in $0<|t|<R$ as $i \rightarrow \infty$, then $u(t) \sim b+(1 / n-1) \log |t|$, as $t \rightarrow 0$, where $b$ is a constant.

Proof. By Lemma 5, there exists $\left\{v_{i}\right\}, i=1,2,3, \ldots$, such that $v_{i}(z)$ is defined in $|z|<R^{1 / n}$, of class $C^{2}$, satisfying (2) there and $v_{i}(z)=u_{i}\left(z^{n}\right)+\log n+(n-1) \log |z|$ in $0<|z|<R^{1 / n}$. Similarly, there exists $\hat{v}_{j}$, such that $\hat{v}_{j}(z)$ is defined in $|z|<R^{1 / n}$, of class $C^{2}$, satisfying (2) there and $\hat{v}_{j}(z)=\hat{u}_{j}\left(z^{n}\right)+\log n+(n-1) \log |z|$ in $0<|z|<R^{1 / n}$.

By assumption, $u_{i} \rightarrow u$ normally in $0<|t|<R$ as $i \rightarrow \infty$. It follows that $v_{i} \rightarrow v$ normally in $0<|z|<R^{1 / n}$, where $v(z)=u\left(z^{n}\right)+\log n+(n-1) \log |z|$. By Lemma 3, $v$ is of class $C^{2}$ and satisfies (2) in $0<|z|<R^{1 / n}$. But $\hat{u}_{1} \leqq u_{i} \leqq \hat{u}_{2}$ in $0<|t|<R$ implies that $\hat{v}_{1} \leqq v_{i} \leqq \hat{v}_{2}$ in $0<|z|<R^{1 / n}$, so that $\hat{v}_{1} \leqq v \leqq \hat{v}_{2}$ in $0<|z|<R^{1 / n}$. Since $\hat{v}_{j}$ is of class $C^{2}$ in $|z|<R^{1 / n}, v$ is bounded in $0<|z|<R^{\prime}<R^{1 / n}$. By Lemma $4, v$ is of class $C^{2}$ in $|z|<R^{\prime}$, hence in $|z|<R^{1 / n}$.

From $v(z)=u\left(z^{n}\right)+\log n+(n-1) \log |z|$, we conclude that $u(t) \sim v(0)-\log n$ $+(1 / n-1) \log |t|=b+(1 / n-1) \log |t|$, as $t \rightarrow 0$, where $b$ is a constant.

Corollary. If $\left\{\lambda_{i}\right\}, i=1,2,3, \ldots,\left\{\hat{\lambda}_{j}\right\}, j=1,2$, are positive $C^{2}$ solutions of (1) in $0<|t|<R, \hat{\lambda}_{1} \leqq \lambda_{i} \leqq \hat{\lambda}_{2}$ in $0<|t|<R$ for all $i, \lambda_{i}(t) \sim a_{i}|t|^{1 / n-1}, \hat{\lambda}_{j} \sim \hat{a}_{j}|t|^{1 / n-1}$, as $t \rightarrow 0$, where $a_{i}, \hat{a}_{j}>0, n$ a positive integer and, finally, $\lambda_{i} \rightarrow \lambda$ normally in $0<|t|<R$ as $i \rightarrow \infty$, then $\lambda(t) \sim a|t|^{1 / n-1}$ as $t \rightarrow 0$, where $a>0$ is a constant.

III. The uniqueness of the Poincaré metric. This section consists of two parts. In the first we describe the behavior on a Riemann surface with signature, of the metric which is induced from the Poincaré metric on the unit disk. In the second part we show that this metric is uniquely determined by this behavior.

Let $(S, \boldsymbol{P}, \boldsymbol{v})$ be a nonexceptional Riemann surface with signature. Let it be represented by a Fuchsian group $G$. Let $g: \hat{\Delta}_{G} \rightarrow S$ be the natural projection.

The Poincaré metric on $\Delta$ is defined by the element of arc length $d s=\lambda(z)|d z|$ $=\left(1 /\left(1-|z|^{2}\right)\right)|d z|, z \in \Delta$. It has constant Gaussian curvature -4 , and $\Delta$ together with this metric is a complete metric space. It can be transferred to $S^{b}$. Indeed, $g: \Delta \rightarrow S^{\#}$ is locally $1-1$ at each point of $\Delta_{G}$, and we can define a metric on $S^{b}$ by the element of arc length $\lambda_{S}(t)|d t|=\lambda(z)|d z|$, with $t=g(z)$, where $t$ is a local parameter on $S$. This Poincaré metric on $S^{b}$ is independent of the choice of the representing Fuchsian group $G$ of $(S, P, v)$. Also, from the conformal invariance 
of the Poincare metric, we may use the upper half-plane $U$ instead of $\Delta$ to describe this Poincaré metric on $S^{b}$.

Since $g$ is a local isometry from $\Delta_{G}$ onto $S^{b}$, the Poincaré metric-on $S^{b}$ has Gaussian curvature -4 . Since the Gaussian curvature is given by $K$ $=-\left(4 / \lambda_{S}^{2}(t)\right)\left(\partial^{2} / \partial t \partial \bar{t}\right) \log \lambda_{S}(t)$, it follows that $\lambda_{S}(t)$ satisfies (1).

We shall next study the behavior of the Poincaré metric near points $P_{k}$ with $2 \leqq v_{k}$.

(i) Near a point $P_{k}$ with $2 \leqq v_{k}<\infty$. Let $P_{k}$ be the image of a point $z_{0} \in \Delta$, then $z_{0}$ is an elliptic fixed point of order $\nu_{k}$. We may assume that $z_{0}=0$. In a neighborhood of $P_{k}$, there is a local parameter $t\left(P_{k}\right.$ corresponds to $\left.t=0\right)$ on $S$ such that $t=g(z)$ can be expressed as $t=z^{v_{k}}$ in a neighborhood of $z_{0}=0$. It follows that $\lambda_{S}(t)$ $\sim b|t|^{1 / v_{k}-1}$, as $t \rightarrow 0$, where $b$ is a positive constant depending on the choice of the local parameter $t$. If we use any other local parameter $\hat{t}$ on $S$ which is 0 for $t=0$, we have $\hat{\lambda}_{S}(\hat{t}) \sim \hat{b}|\hat{t}|^{1 / v_{k}-1}$, as $\hat{t} \rightarrow 0$, where $\hat{\lambda}_{S}(\hat{t})|d \hat{t}|=\lambda_{S}(t)|d t|$, and $\hat{b}$ is a positive constant.

(ii) Near a point $P_{k}$ with $\nu_{k}=\infty$. Consider $U$ instead of $\Delta$. Let $P_{k}$ be the image of a point $z_{0}$. Then $z_{0}$ is a parabolic fixed point. We may assume that $z_{0}=\infty$. Then there is a local parameter $t\left(t=0\right.$ at $\left.P_{k}\right)$ such that $t=g(z)=\exp (2 \pi i z / d), d>0$, in a neighborhood of $z_{0}=\infty$. Note that the Poincaré metric on $U$ is given by $\lambda(z)|d z|$ $=(i /(z-\bar{z}))|d z|, z \in U$. Hence in a neighborhood of $P_{k}, \lambda_{S}(t) \sim-1 /(2|t| \log |t|)$, as $t \rightarrow 0$. If we use another local parameter $\hat{t}$ on $S$ which is 0 for $t=0$, we have again $\hat{\lambda}_{S}(\hat{t}) \sim-1 /(2|\hat{t}| \log |\hat{t}|)$, as $\hat{t} \rightarrow 0$, where $\hat{\lambda}_{S}(\hat{t})|d \hat{t}|=\lambda_{S}(t)|d t|$.

In the Poincaré metric on $\Delta$, any (sufficiently smooth) curve leading to the boundary has infinite length. $g$ preserves arc length. Therefore any (sufficiently smooth) curve beginning at a point on $S$ other than points $P_{k}$ with $v_{k}=\infty$ and leading to a point $P_{k}$ with $\nu_{k}=\infty$ or to the ideal boundary of $S$ has infinite length (measured by integrating $\left.\lambda_{S}(t)|d t|\right)$. The definition and properties of the ideal boundary can be found in Ahlfors and Sario [1, Chapter I, \$13 A].

From now on, by abuse of language, we shall not distinguish between the arc length element and the metric induced by it.

The following lemma is a well-known result.

Lemma 7. (i) Let $\lambda(z)|d z|$ be the Poincaré metric on $\Delta$.

(ii) Let $\sigma(z)|d z|$ be another metric defined on $\Delta$, with $\sigma(z)$ of class $C^{2}$, and with Gaussian curvature -4. Assume also that $\Delta$ with the metric $\sigma(z)|d z|$ is complete.

Then $\sigma(z)=\lambda(z)$.

THEOREM 2. Let $(S, P, v)$ be a nonexceptional Riemann surface with signature. Let it be represented by a Fuchsian group $G$. Let $\lambda_{S}(t)|d t|$ be the Poincaré metric on $S^{b}$ defined by $G$. Let $\sigma_{S}(t)|d t|$ be another metric defined on $S^{b}$, with $\sigma_{S}(t)$ of class $C^{2}$. At any point $P_{k}$ with $2 \leqq \nu_{k}<\infty$, and for all local parameter $t$ with $t=0$ at $P_{k}$, let $\sigma_{S}(t) \sim d|t|^{1 / v_{k}-1}$ as $t \rightarrow 0$, where $d$ is a positive constant depending on $P_{k}$ and on the choice of the local parameter $t$. Let the metric $\sigma_{S}(t)|d t|$ have constant Gaussian 
curvature -4. Finally, assume that in the metric $\sigma_{S}(t)|d t|$ any (sufficiently smooth) curve on $S$ beginning at a point other than $P_{k}$ with $\nu_{k}=\infty$ and leading to a point $P_{k}$ with $\nu_{k}=\infty$ or to the ideal boundary of $S$ has infinite length.

Then the two metrics coincide: $\sigma_{S}(t)=\lambda_{S}(t)$, where $t$ is any local parameter.

Proof. Let $g: \Delta \rightarrow S^{\#}$ be the natural projection. By assumption $\lambda_{S}(t)|d t|$ $=\lambda(z)|d z|, t=g(z)$, where $\lambda(z)|d z|$ is the Poincaré metric on $\Delta$. Lift $\sigma_{S}(t)|d t|$ to a metric $\sigma(z)|d z|$ on $\Delta_{G}$ by $g$, so that $\sigma_{S}(t)|d t|=\sigma(z)|d z|, t=g(z)$. Hence $\sigma_{S}(t) / \lambda_{S}(t)$ $=\sigma(z) / \lambda(z), t=g(z)$. By assumption, at any point $P_{k}$ with $2 \leqq \nu_{k}<\infty$, and in any local parameter $t$ with $t=0$ at $P_{k}$, we have $\lim _{t \rightarrow 0}\left(\sigma_{S}(t) / \lambda_{S}(t)\right)=c$, where $c$ is a positive constant depending on the point $P_{k}$ only.

Also $\sigma(z)$ is of class $C^{2}$ in $\Delta_{G}$. By what was just said, at an elliptic fixed point $z_{0}$ of $G$ in $\Delta$, we have $\lim _{z \rightarrow z_{0}}(\sigma(z) / \lambda(z))=c$, where $c$ is a positive constant depending on the point $z_{0}$. Hence $\sigma(z)$ is continuous and positive in $\Delta$.

Since $\sigma_{S}(t)|d t|$ has Gaussian curvature -4 on $S^{b}, \sigma(z)|d z|$ has Gaussian curvature -4 on $\Delta_{G}$. Therefore $\sigma(z)$ satisfies (1) in $\Delta_{G}$. Consider a relatively compact domain $D$ in $\Delta$ containing an elliptic fixed point $z_{0}$ of $G$ and no others in its interior. Since $\sigma(z)$ is continuous and positive in $\Delta, \log \sigma(z)$ is continuous in $\Delta$ and hence bounded in $D$. By Lemma $4, \log \sigma(z)$, hence $\sigma(z)$, is of class $C^{2}$ in $D$ and $\sigma(z)$ satisfies (1) in $D$. The same argument applies to all other elliptic fixed points in $\Delta$, so that $\sigma(z)$ is of class $C^{2}$ in $\Delta$ and satisfies (1) in $\Delta$. It follows that the metric $\sigma(z)|d z|$ is defined on $\Delta$ and has constant Gaussian curvature -4 .

Since $g$ preserves arc length, it follows from the assumption that any (sufficiently smooth) curve from an interior point to the boundary of $\Delta$ in $\sigma(z)|d z|$ has infinite length. Therefore, $\Delta$ together with the metric $\sigma(z)|d z|$ is a complete metric space. By Lemma 7, $\sigma(z)=\lambda(z)$ on $\Delta$. This implies the conclusion of the theorem.

IV. The approximation theorem for the metric. Given a nonexceptional Riemann surface with signature $(S, \boldsymbol{P}, \boldsymbol{\nu})$, each subdomain of $S$ may be considered, in an obvious way, as a Riemann surface with signature. We can choose a sequence $\left\{S_{i}\right\}, i=1,2,3, \ldots$, of subdomains of $S$ with the following properties: (i) $S_{1} \subset \subset S_{2}$ $\subset \subset \ldots \subset \subset S$, where $\subset \subset$ means "relatively compact", (ii) $S=\bigcup_{i=1}^{\infty} S_{i}$, (iii) each $S_{i}$ is a Riemann surface of finite type and contains at most a finite number of points $P_{k}$, (iv) the boundary of each $S_{i}$ consists of a finite number of simple closed analytic curves which do not pass through any of the points $P_{k}$. For $i$ large, the Riemann surface with signature $\left(S_{i}, \boldsymbol{P}, \boldsymbol{\nu}\right)$ is nonexceptional; here and hereafter $\left(S_{i}, \boldsymbol{P}, \boldsymbol{v}\right)$ means $S_{i}$ together with those points $P_{k} \in S_{i}$ and their associated "integers" $\nu_{k}$.

We assume now that $\left(S_{i}, \boldsymbol{P}, \nu\right)$ is nonexceptional for $i=1,2,3, \ldots$ We can then represent $\left(S_{i}, \boldsymbol{P}, \nu\right)$ by a Fuchsian group $G_{i}$, and $S_{i}$ carries a Poincaré metric defined by $G_{i}$. Denote it by $\lambda_{i}(t)|d t|$. On the other hand, we shall write $\lambda(t)|d t|$ for $\lambda_{S}(t)|d t|$, the Poincaré metric on $S$ induced by the group $G$ which represents $(S, \boldsymbol{P}, \boldsymbol{v})$. 
THEOREM 3. Under the circumstances described above, we have

(i) $\lambda_{i}(t) \geqq \lambda_{i+1}(t), \lambda_{i}(t) \geqq \lambda(t)$ on $S_{i}^{b}=S_{i}-\bigcup_{v_{k} \geqq 2}\left\{P_{k}\right\}$, and

(ii) $\lambda_{i}(t) \rightarrow \lambda(t)$ normally on $S^{b}=S-\bigcup_{v_{k} \geqq_{2}}\left\{P_{k}\right\}$, as $i \rightarrow \infty$.

(Here $t$ is the same local parameter for all $\lambda_{i}$ and $\lambda_{\text {.) }}$

Proof. (i) Recall that $\lambda$ and $\lambda_{i}$ satisfy (1).

On $S_{i}^{b}$, set

$$
\delta(t)=u_{i+1}(t)-u_{i}(t)=\log \lambda_{i+1}(t)-\log \lambda_{i}(t)=\log \left(\lambda_{i+1}(t) / \lambda_{i}(t)\right) .
$$

Note that $\delta(t)$ is invariant under local parameter change, hence $\delta$ is a function on $S_{i}^{b}$. In any parametric disk, both $u_{i+1}$ and $u_{i}$ satisfy (2), implying that $\delta$ has neither positive maxima nor negative minima in the neighborhood considered (Lemma 1), and hence anywhere on $S_{i}^{b}$.

We shall now study the behavior of $\delta$ at the points $P_{k}$ with $\nu_{k} \geqq 2$ on $S_{i}$. We claim that $\delta$ is continuous at any point $P_{k}$ with $2 \leqq v_{k}<\infty$ and has neither a positive maximum nor a negative minimum there. Recall that $\lambda_{t+1}(t) \sim b_{1}|t|^{1 / v_{k}-1}, \lambda_{t}(t)$ $\sim b_{0}|t|^{1 / v_{k}-1}$, as $t \rightarrow 0$ (as usual, $P_{k}$ corresponds to $t=0$ ), where $b_{1}, b_{0}$ are positive constants depending on the choice of the local parameter. Therefore $\delta=$ $\log \left(\lambda_{i+1}(t) / \lambda_{t}(t)\right)$ is continuous at $P_{k}$. By the corollary of Lemma 5 , there exist functions $\hat{\lambda}_{i+1}(z), \hat{\lambda}_{i}(z)$, of class $C^{2}$, satisfying (1) in a neighborhood of $z=0$, and $\hat{\lambda}_{i+1}(z)|d z|=\lambda_{i+1}(t)|d t|, \hat{\lambda}_{i}(z)|d z|=\lambda_{i}(t)|d t|$, with $t=z^{v_{k}}$, in a deleted neighborhood of $z=0$. Consider $\delta$ as a function of $z: \delta=\log \hat{\lambda}_{i+1}(z)-\log \hat{\lambda}_{i}(z)$. By Lemma $1, \delta$ has neither positive maxima nor negative minima in the neighborhood considered. Thus $\delta$ has neither a positive maximum nor a negative minimum at $\boldsymbol{P}_{\boldsymbol{k}}$.

At a point $P_{k}$ with $v_{k}=\infty$, we claim that $\delta$ is continuous and $\delta=0$. In $\S I I I$ we showed that the asymptotic behavior of the Poincaré metric near a point $P_{k}$ with $\nu_{k}=\infty$ is always given by $-1 /(2|t| \log |t|)$, as $t \rightarrow 0$. $\left(P_{k}\right.$ corresponds to $t=0$.) Thus $\delta \sim \log 1=0$, as $t \rightarrow 0$.

We therefore conclude that $\delta$ has neither positive maxima nor negative minima not only on $S_{i}^{b}$ but also on $S_{i}$.

Next we claim that $\delta \leqq 0$ on $S_{i}$. For this purpose, we have to examine the behavior of $\delta$ on the boundary of $S_{i}$. The boundary of $S_{i}$ consists of a finite number of simple closed analytic curves $C_{1}, \ldots, C_{r}$. They lie in the interior of $S_{i+1}$ and do not pass through any of the points $P_{k}, k=1,2,3, \ldots$ Let us consider $U$ instead of $\Delta$. Let $Q$ be a point of $C_{j}$. Then a preimage of $Q$ is a point $z_{0} \in \boldsymbol{R}$. In a neighborhood of $z_{0}$, the natural projection from $U$ onto $S_{i}$ can be expressed as $t=z-z_{0}$. Hence $\lambda_{i}(t)=(i /(z-\bar{z}))|d z / d t| \sim \infty$, as $t \rightarrow 0$. On the other hand, $Q$ is an interior point of $S_{i+1}$, and $Q \neq P_{k}, k=1,2,3, \ldots$, so that $\lambda_{i+1}(t)$ is defined at $Q$. It follows that $\delta \sim-\infty$, as $t \rightarrow 0$. A similar argument applies to all points of $C_{1}, C_{2}, \ldots, C_{r}$. It follows easily that $\delta \leqq 0$ on $S_{i}$, so that $\lambda_{i+1}(t) \leqq \lambda_{i}(t)$ on $S_{i}^{b}$.

With $S_{\imath} \subset \subset S$, we apply the same argument to the metrics $\lambda_{i}(t)|d t|$ and $\lambda(t)|d t|$ and conclude in exactly the same way that $\lambda(t) \leqq \lambda_{t}(t)$ on $S_{i}^{b}$. 
(ii) It follows from part (i) that the metrics considered converge in the following sense: if $D$ is a relatively compact domain in $S^{b}$ with a local parameter $t$ defined in $D$, then $\lim _{i \rightarrow \infty} \lambda_{i}(t)=\sigma(t)$ exists on $D$. (Note that $\lambda_{i}(t)$ will be defined for $i$ sufficiently large, since $D \subset S_{i}^{b}$ for large $i$.) It is clear that $\sigma(t)|d t|$ is independent of the choice of local parameter.

We show next that the convergence $\lambda_{i} \rightarrow \sigma$ is normal. More precisely, if $D$ is as before, and $D_{0} \subset \subset D$, then $\lambda_{i}(t) \rightarrow \sigma(t)$ uniformly in $D_{0}$ as $i \rightarrow \infty$. From part (i), we have $\lambda(t) \leqq \lambda_{i}(t) \leqq \lambda_{i_{0}}(t)$ for all $i \geqq i_{0}$, where $i_{0}$ is chosen such that $\lambda_{i}, i \geqq i_{0}$, is defined in $D$. Thus $\log \lambda(t) \leqq \log \lambda_{i}(t) \leqq \log \lambda_{i_{0}}(t)$ in $D$, so that $\left|\log \lambda_{i}(t)\right| \leqq M$ for $i \geqq i_{0}$. By Lemma 2 , we conclude that any infinite sequence of the family $\left\{\log \lambda_{i}\right\}$ has a uniformly convergent subsequence in $D_{0}$. The same is true for the family $\left\{\lambda_{i}\right\}$. But $\lambda_{i} \rightarrow \sigma$ in $D$ as $i \rightarrow \infty$, it follows that the convergence is uniform in $D_{0}$.

Furthermore, by Lemma 3 we conclude that $\log \sigma(t)$ is of class $C^{2}$, hence $\sigma(t)$ is positive, of class $C^{2}$, and $\sigma(t)$ satisfies (1) in $D$. In conclusion, $\sigma(t)|d t|$ defines a metric on $S^{b}$, which has constant Gaussian curvature -4 .

We shall show that near any point $P_{k}$ with $2 \leqq v_{k}<\infty, \sigma(t) \sim d|t|^{1 / v_{k}-1}$, as $t \rightarrow 0$, where $d$ is a positive constant. (Here $P_{k}$ corresponds to $t=0$.) Let $D$ be a relatively compact domain in $S$ containing $P_{k}$ in interior and with a local parameter $t$ defined in it. $\left(P_{k}\right.$ corresponds to $t=0$.) Also assume that $D$ contains no other points $P_{k}$ with $2 \leqq v_{k}$. Then there exists $i_{0}$ such that, for $i \geqq i_{0}, \lambda_{i}$ is defined in $D-\left\{P_{k}\right\}$. By part (i), we have $\lambda(t) \leqq \lambda_{i}(t) \leqq \lambda_{i_{0}}(t)$ in $D-\left\{P_{k}\right\}$. Since $\lambda(t) \sim b|t|^{1 / v_{k}-1}, \lambda_{i}(t)$ $\sim b_{i}|t|^{1 / v_{k}-1}$, as $t \rightarrow 0$, where $b, b_{i}$ are positive constants and $i \geqq i_{0}$, and $\lambda_{i} \rightarrow \sigma$ normally in $D-\left\{P_{k}\right\}$ as $i \rightarrow \infty$, we conclude from the corollary of Lemma 6 that $\sigma(t) \sim d|t|^{1 / v_{k}-1}$, as $t \rightarrow 0$, where $d$ is a positive constant.

Finally, from part (i) and the fact that $\lambda_{i} \rightarrow \sigma$ as $i \rightarrow \infty$, we have $\lambda(t) \leqq \sigma(t)$ on $S^{b}$. For any (sufficiently smooth) curve $\gamma$ from a point of $S$ other than $P_{k}$ with $\nu_{k}=\infty$ to a point $P_{k}$ with $\nu_{k}=\infty$ or to the ideal boundary of $S, \int_{\gamma} \lambda(t)|d t|=\infty$. Therefore $\int_{\gamma} \sigma(t)|d t|=\infty$. By Theorem 2, $\sigma(t)=\lambda(t)$ on $S$. This implies the conclusion of part (ii).

\section{The monotonicity theorem for the metric.}

Definition 3. Given $\nu^{\prime}=\left\{v_{k}^{\prime}\right\}, \nu^{\prime \prime}=\left\{\nu_{k}^{\prime \prime}\right\}$, we define $\nu^{\prime} \leqq \nu^{\prime \prime}$ if and only if $\nu_{k}^{\prime} \leqq \nu_{k}^{\prime \prime}$ for all $k$.

THEOREM 4. Given $S, P, \nu^{\prime}, \nu^{\prime \prime}$ such that $\nu^{\prime} \leqq \nu^{\prime \prime}$. Assume that the Riemann surfaces with signature $\left(S, P, v^{\prime}\right)$ and $\left(S, P, v^{\prime \prime}\right)$ are nonexceptional. Let $\lambda^{\prime}(t)|d t|, \lambda^{\prime \prime}(t)|d t|$ be the Poincaré metrics on $S$, defined by the Fuchsian groups $G^{\prime}, G^{\prime \prime}$ which represent $\left(S, P, v^{\prime}\right),\left(S, P, \nu^{\prime \prime}\right)$ respectively. Then $\lambda^{\prime}(t) \leqq \lambda^{\prime \prime}(t)$ on $S^{*}=S-\bigcup_{v_{k}^{\prime} \text { or } v_{k}^{*} \geqq 2}\left\{P_{k}\right\}$. ( $t$ is the same local parameter.)

Proof. As in $\S I V$, we choose a sequence $\left\{S_{i}\right\}$ of subdomains of $S$ with the described properties. We may assume that $\left(S_{i}, \boldsymbol{P}, \boldsymbol{\nu}^{\prime}\right)$ and $\left(S_{i}, \boldsymbol{P}, \boldsymbol{\nu}^{\prime \prime}\right)$ are not exceptional 
for $i=1,2,3, \ldots$ We can represent $\left(S_{i}, \boldsymbol{P}, \boldsymbol{\nu}^{\prime}\right)$ by a Fuchsian group $G_{i}^{\prime}$ so that $S_{i}$ carries a Poincaré metric defined by $G_{i}^{\prime}$. Denote it by $\lambda_{i}^{\prime}(t)|d t|$. Similarly, we can represent $\left(S_{i}, \boldsymbol{P}, \boldsymbol{\nu}^{\prime \prime}\right)$ by a Fuchsian group $G_{i}^{\prime \prime}$ so that $S_{i}$ carries a Poincaré metric induced by $G_{i}^{\prime \prime}$. Denote it by $\lambda_{i}^{\prime \prime}(t)|d t|$.

By Theorem 3, $\lambda_{i}^{\prime}(t) \rightarrow \lambda^{\prime}(t)$ as $i \rightarrow \infty$ on $S-\bigcup_{v_{k}^{\prime} \geqq 2}\left\{P_{k}\right\}$ and $\lambda_{i}^{\prime \prime}(t) \rightarrow \lambda^{\prime \prime}(t)$ as $i \rightarrow \infty$ on $S-\bigcup_{v_{k}^{*} \geqq 2}\left\{P_{k}\right\}$. We shall prove that $\lambda_{i}^{\prime}(t) \leqq \lambda_{i}^{\prime \prime}(t)$ on $S_{i}^{*}=S_{i}$ $-\bigcup_{v_{k}^{\prime} \text { or } v_{k}^{*} \geqq 2}\left\{P_{k}\right\}$; this will imply the assertion. On $S_{i}^{*}$ set $\delta(t)=\log \lambda_{i}^{\prime}(t)-\log \lambda_{i}^{\prime \prime}(t)$. Then as in $\S I V$, we can show that $\delta$ is a function defined on $S_{i}^{*}$ and has neither positive maxima nor negative minima there. To show $\delta \leqq 0$ on $S_{i}^{*}$, we shall study the behavior of $\delta$ at the points $P_{k}$ with either $\nu_{k}^{\prime}$ or $\nu_{k}^{\prime \prime} \geqq 2$ on $S_{i}$ and also on the boundary of $S_{i}$.

Case (i). At a point $P_{k}$ with $2 \leqq \nu_{k}^{\prime}=\nu_{k}^{\prime \prime}<\infty, \delta$ is continuous and has neither a positive maximum nor a negative minimum.

Case (ii). At a point $P_{k}$ with $\nu_{k}^{\prime}=\nu_{k}^{\prime \prime}=\infty, \delta$ is continuous and $\delta=0$ there.

Case (iii). At a point $P_{k}$ with $\nu_{k}^{\prime} \neq \nu_{k}^{\prime \prime}$ (hence $1 \leqq v_{k}^{\prime}<\nu_{k}^{\prime \prime}$ ), we claim that $\delta \sim-\infty$ as $t \rightarrow 0$, where $t=0$ corresponds to $P_{k}$. Indeed, if $\nu_{k}^{\prime}=1$, then $\lambda_{i}^{\prime}(t)$ is continuous at $t=0$. And $\lambda_{i}^{\prime \prime}(t) \sim b^{\prime \prime}|t|^{1 / v_{k}^{\prime \prime}-1}$ or $\lambda_{i}^{\prime \prime}(t) \sim-1 /(2|t| \log |t|)$, as $t \rightarrow 0$ according as $\nu_{k}^{\prime \prime}<\infty$ or $\nu_{k}^{\prime \prime}=\infty$, where $b^{\prime \prime}$ is a positive constant. In both cases, $\delta \sim-\infty$ as $t \rightarrow 0$. If $2 \leqq v_{k}^{\prime}<\nu_{k}^{\prime \prime}<\infty$, we have $\lambda_{i}^{\prime}(t) \sim b^{\prime}|t|^{1 / v_{k}^{\prime}-1}$, and $\lambda_{i}^{\prime \prime}(t) \sim b^{\prime \prime}|t|^{1 / v_{k}^{\prime}-1}$, as $t \rightarrow 0$, where $b^{\prime}, b^{\prime \prime}$ are positive constants, so that $\delta \sim-\infty$ as $t \rightarrow 0$. Finally, if $2 \leqq v_{k}^{\prime}<\nu_{k}^{\prime \prime}=\infty$, we have $\lambda_{i}^{\prime}(t) \sim b^{\prime}|t|^{1 / v_{k}^{\prime \prime}-1}, \lambda_{i}^{\prime \prime}(t) \sim-1 /(2|t| \log |t|)$, as $t \rightarrow 0$. By direct calculation, it follows that $\delta \sim-\infty$ as $t \rightarrow 0$.

Case (iv). On the boundary of $S_{i}$, we claim that $\delta$ is continuous and $\delta=0$. As before, denote the boundary curves by $C_{1}, \ldots, C_{r}$. Let $Q$ be a point on $C_{j}$. Consider $U$ instead of $\Delta . \lambda_{i}^{\prime}(t)|d t|, \lambda_{i}^{\prime \prime}(t)|d t|$ come from the Poincaré metric in $U$ via two different natural projections. Let $z_{0} \in \boldsymbol{R}$ be a preimage of $Q$ in the complex $z$-plane under one of the natural projections. Let $w_{0} \in \boldsymbol{R}$ be a preimage of $Q$ in the complex $w$-plane under the other natural projection.

There exists a conformal mappings of a (half) neighborhood of $w_{0}$ in the upper half $w$-plane onto a (half) neighborhood of $z_{0}$ in the upper half $z$-plane. Furthermore, it sends a segment of $\boldsymbol{R}$ onto $\boldsymbol{R}$. By the reflection principle, it can be extended to a conformal mapping of a full neighborhood of $w_{0}$ onto a full neighborhood of $z_{0}$. Expressing it in power series, we have $z-z_{0}=a_{1}\left(w-w_{0}\right)+a_{2}\left(w-w_{0}\right)^{2}+\cdots$, where $a_{1}>0$, and all coefficients are real.

On the other hand, $\lambda_{i}^{\prime}(t)|d t|=(i /(z-\bar{z}))|d z|$, and $\lambda_{i}^{\prime \prime}(t)|d t|=(i /(w-\bar{w}))|d w|$, so that $\lambda_{i}^{\prime}(t) / \lambda_{i}^{\prime \prime}(t) \sim\left(1 / a_{1}\right) a_{1}=1$, as $t \rightarrow 0$. Thus $\delta=0$ at $Q$. Similar considerations show that $\delta=0$ on all curves $C_{1}, \ldots, C_{r}$.

It follows easily that $\delta \leqq 0$ on $S_{i}^{*}$, so that $\lambda_{i}^{\prime}(t) \leqq \lambda_{i}^{\prime \prime}(t)$ on $S_{i}^{*}$.

\section{The limit theorem for the metric.}

Definition 4. Given $\nu^{j}=\left\{\nu_{k}^{j}\right\}, j=1,2,3, \ldots$, and $\nu=\left\{\nu_{k}\right\}$, we define $\nu^{j} \rightarrow \nu$, as $j \rightarrow \infty$, to mean that $\nu_{k}^{j} \rightarrow \nu_{k}$, as $j \rightarrow \infty$, for all $k$. 
THEOREM 5. Given $S, P, \nu^{0}, \nu, \nu^{j}, j=1,2,3, \ldots$, such that $\nu^{0} \leqq \nu^{j}$ for all $j$ and $\nu^{j} \rightarrow \nu$, as $j \rightarrow \infty$. Assume that $\left(S, P, v^{0}\right),(S, P, v),\left(S, P, v^{j}\right), j=1,2,3, \ldots$, are all nonexceptional Riemann surfaces with signature. Let $\lambda_{0}(t)|d t|, \lambda(t)|d t|, \lambda_{j}(t)|d t|$, $j=1,2,3, \ldots$, be the Poincaré metrics on $S$, induced by the Fuchsian groups $G_{0}, G, G_{j}$, $j=1,2,3, \ldots$, which represent $\left(S, P, \nu^{0}\right),(S, P, \nu),\left(S, P, \nu^{j}\right), j=1,2,3, \ldots$, respectively.

Then $\lambda_{j}(t) \rightarrow \lambda(t)$ normally on $S^{b}=S-\bigcup_{v_{k} \geqq 2}\left\{P_{k}\right\}$, as $j \rightarrow \infty$. (Here $t$ is the same local parameter for all $\lambda_{j}$ and $\lambda_{\text {.) }}$

Proof. Define $\nu^{\infty}=\left\{\nu_{k}^{\infty}\right\}$ with $\nu_{k}^{\infty}=\infty$ for all $k .\left(S, P, \nu^{\infty}\right)$ is then a nonexceptional Riemann surface with signature. Let $\lambda_{\infty}(t)|d t|$ be the Poincaré metric on $S$, defined by the Fuchsian group $G_{\infty}$ which represents $\left(S, P, \nu^{\infty}\right)$. Let $D$ be a parametric disk in $S_{0}=S-\left\{\right.$ all points $\left.P_{k}\right\}$ with local parameter $t$. Since $\boldsymbol{\nu}^{0} \leqq \boldsymbol{\nu}^{j} \leqq \boldsymbol{\nu}^{\infty}$, by Theorem 4, $\lambda_{0}(t) \leqq \lambda_{j}(t) \leqq \lambda_{\infty}(t)$, hence $\log \lambda_{0}(t) \leqq \log \lambda_{j}(t) \leqq \log \lambda_{\infty}(t)$ in $D$. Let $D_{0} \subset \subset D_{1} \subset \subset D$. Then $\left|\log \lambda_{j}(t)\right| \leqq M$ in $D_{1}$, for all $j$. By Lemma 2 , any infinite sequence of the family $\left\{\log \lambda_{j}\right\}, j=1,2,3, \ldots$, has a uniformly convergent subsequence in $D_{0}$. The same is true for the family $\left\{\lambda_{j}\right\}, j=1,2,3, \ldots$

Consider a point $P=P_{k}$ with $\nu_{k}=1 . P \in S^{b}$. (But $P \notin S_{0}$.) Let $D$ be a parametric disk in $S^{b}$ with local parameter $t$ containing $P$ and no other points $P_{k}(t=0$ at $P$ ). Let $P \in D_{0} \subset \subset D_{1} \subset \subset D$. By assumption, $\nu_{k}^{j} \rightarrow \nu_{k}=1$ as $j \rightarrow \infty$, hence there exists $j_{0}$ such that $v_{k}^{\prime}=1$ for $j \geqq j_{0}$. Define $\nu^{\prime}=\left\{v_{n}^{\prime}\right\}$ with $\nu_{n}^{\prime}=\infty$ for $n \neq k, v_{n}^{\prime}=1$ for $n=k$. Then $\left(S, P, \nu^{\prime}\right)$ is a nonexceptional Riemann surface with signature. Let $\lambda^{\prime}(t)|d t|$ be the Poincaré metric on $S$, defined by the Fuchsian group $G^{\prime}$ which represents $\left(S, P, \nu^{\prime}\right)$. Since $\nu^{0} \leqq \nu^{j} \leqq \nu^{\prime}$, for $j \geqq j_{0}$, by Theorem $4, \lambda_{0}(t) \leqq \lambda_{j}(t) \leqq \lambda^{\prime}(t)$, hence $\log \lambda_{0}(t)$ $\leqq \log \lambda_{j}(t) \leqq \log \lambda^{\prime}(t)$ in $D$, so that $\left|\log \lambda_{j}(t)\right| \leqq M$ in $D_{1}$. By Lemma 2 again, any infinite sequence of the family $\left\{\log \lambda_{j}\right\}, j \geqq j_{0}$, has a uniformly convergent subsequence in $D_{0}$. The same is clearly true for the family $\left\{\log \lambda_{j}\right\}, j=1,2,3, \ldots$, hence for the family $\left\{\lambda_{j}\right\}, j=1,2,3, \ldots$, also.

Since every Riemann surface has a countable base, $S^{b}$ can be covered by a countable number of domains like $D_{0}$ described above. By the usual diagonal method, one sees that, on $S^{b}$, any infinite sequence of the family $\left\{\lambda_{j}\right\}, j=1,2,3, \ldots$, has a normally convergent subsequence. Let $\left\{\lambda_{j_{k}}\right\}$ be such a subsequence and $\lambda_{j_{k}}(t) \rightarrow \sigma(t)$ as $j_{k} \rightarrow \infty$. We shall prove that $\sigma(t)=\lambda(t)$ on $S^{b}$. This will imply that the original sequence $\left\{\lambda_{j}\right\}, j=1,2,3, \ldots$, converges to $\lambda$ normally on $S^{b}$, as $j \rightarrow \infty$. For simplicity, we may assume that $\lambda_{f}(t) \rightarrow \sigma(t)$ normally on $S^{b}$, as $j \rightarrow \infty$, and show that $\sigma(t)=\lambda(t)$.

By Lemma 3, we conclude that $\log \sigma(t)$ is of class $C^{2}$, hence $\sigma(t)$ is positive and of class $C^{2}$, and $\sigma(t)$ satisfies (1) in $D_{0}$. Clearly $\sigma(t)|d t|$ is invariant, so that it defines a metric on $S^{b}$. It has constant Gaussian curvature -4 on $S^{b}$. (Compare §IV.) We shall show that near any point $P_{k}$ with $2 \leqq \nu_{k}<\infty, \sigma(t) \sim d|t|^{1 / v_{k}-1}$, as $t \rightarrow 0$, where $d$ is a positive constant. (Here $P_{k}$ corresponds to $t=0$.) Let $D$ be a parametric disk in $S$ with local parameter $t$, containing $P_{k}\left(P_{k}\right.$ corresponds to $\left.t=0\right)$ 
and no other points $P_{j}, j \neq k$, in its interior. By assumption, $\nu_{k}^{j} \rightarrow \nu_{k}$ as $j \rightarrow \infty$. Since $\nu_{k}<\infty$, there exists $j_{0}$ such that $\nu_{k}^{j}=\nu_{k}$ for $j \geqq j_{0}$. Define $\nu^{\prime \prime}=\left\{\nu_{n}^{\prime \prime}\right\}$ with $\nu_{n}^{\prime \prime}=\infty$ for $n \neq k, \nu_{n}^{\prime \prime}=v_{k}$ for $n=k$. Then $\left(S, \boldsymbol{P}, \nu^{\prime \prime}\right)$ is a nonexceptional Riemann surface with signature. Let $\lambda^{\prime \prime}(t)|d t|$ be the Poincare metric on $S$, defined by the Fuchsian group $G^{\prime \prime}$ which represents $\left(S, P, \nu^{\prime \prime}\right)$. Since $\nu^{0} \leqq \nu^{j} \leqq \nu^{\prime \prime}$, for $j \geqq j_{0}$, by Theorem $4, \lambda_{0}(t)$ $\leqq \lambda_{j}(t) \leqq \lambda^{\prime \prime}(t)$ in $D-\left\{P_{k}\right\}$. Since $\lambda_{j} \rightarrow \sigma$ normally in $D-\left\{P_{k}\right\}$ as $j \rightarrow \infty$, we conclude from the corollary of Lemma 6 that $\sigma(t) \sim d|t|^{1 / v_{k}-1}$ as $t \rightarrow 0$, where $d$ is a positive constant.

Finally, we shall show that if $\gamma$ is a (sufficiently smooth) curve on $S$ beginning at a point $\boldsymbol{P}$ other than $\boldsymbol{P}_{k}$ with $\nu_{k}=\infty$ and leading to a point $\boldsymbol{P}_{k}$ with $\boldsymbol{\nu}_{k}=\infty$ or to the ideal boundary of $S$, then $\int_{\gamma} \sigma(t)|d t|=\infty$. The latter case follows from the fact that $\lambda_{0}(t) \leqq \lambda_{j}(t)$ for every $j$, so that $\lambda_{0}(t) \leqq \sigma(t)$.

In the former case, let $P_{k}$ be a point with $\nu_{k}=\infty$. By assumption, $\nu_{k}^{j} \rightarrow \infty$, as $j \rightarrow \infty$. Let $D$ be a parametric disk in $S$ with local parameter $t$, containing $P_{k}\left(P_{k}\right.$ corresponds to $t=0)$ and no other points $P_{j}, j \neq k$, in its interior. In $D$, draw a circle $L:|t|=R$. We may assume that $R>1$. Also, we may assume that $\gamma$ is a (sufficiently smooth) curve from a point on $|t|=1$ to $t=0$, lying completely in $|t| \leqq 1$. Let us consider a fixed $j$ and let us write $n$ instead of $v_{k}^{j}$. Assume first that $n<\infty$. By the corollary of Lemma 5 , there exists a function $\hat{\lambda}(z)$, of class $C^{2}$, defined for $|z|<R^{1 / n}$, satisfying (1) and such that $\lambda_{j}(t)|d t|=\hat{\lambda}(z)|d z|$, with $t=z^{n}$. By assumption, $\nu^{0} \leqq \nu^{j}$, for all $j$, hence $\lambda_{0}(t) \leqq \lambda_{j}(t)$ in $D-\left\{P_{k}\right\}$, so that $c<\lambda_{j}(t)$ on $|t|=1$ for all $j$, where $c$ is a positive constant independent of $j$. It follows that on $|z|=1$, cn $<\hat{\lambda}(z)$.

We introduce a metric $\tau(z)|d z|$ with

$$
\tau(z)=a /\left(1-a^{2}|z|^{2}\right), \quad|z|<R^{\prime},
$$

where $0<a<1$ is a constant chosen so that $a /\left(1-a^{2}\right)=c n$, that is,

$$
a=\left(\left(4 c^{2} n^{2}+1\right)^{1 / 2}-1\right) / 2 c n, \text { and } R^{\prime}=\min \left(R^{1 / n}, 1 / a\right) \text {. }
$$

Note that $R^{\prime}>1$ and $a \rightarrow 1$ as $n \rightarrow \infty$. Under this choice of $a, \tau(z)=c n<\lambda(z)$ on $|z|=1$. Set $\delta=\log \tau(z)-\log \hat{\lambda}(z)$. Since $\tau(z)$ satisfies (1), we have, by Lemma 1 , that $\delta$ has neither positive maxima nor negative minima in $|z|<R^{\prime}$. But $\delta<0$ on $|z|=1$ by construction. Thus $\delta \leqq 0$ for $|z| \leqq 1$ and we conclude that $\tau(z) \leqq \hat{\lambda}(z)$ for $|z| \leqq 1$.

Observe that $\tau(z)|d z|$ is a Poincaré metric in the disk $|z|<1 / a$. Hence in $|z| \leqq 1$, any geodesic through the point $z=0$ with respect to this metric is a straight line and vice versa. Also, any diameter has the same length in this metric. Now let $\varepsilon$ be a small positive number and let $\gamma_{\varepsilon}$ be the arc of $\gamma$ leading from the initial point of $\gamma$ to the first point on the intersection of $\gamma$ and the circle $|t|=\varepsilon$. The $\operatorname{arc} \gamma_{\varepsilon}$ is the image of an arc $\hat{\gamma}_{\varepsilon}$ under the mapping $z \mapsto z^{n}$. The arc $\hat{\gamma}_{\varepsilon}$ leads from a point on $|z|=1$ to a point on $|z|=\varepsilon^{1 / n}$. We have

$$
\int_{\gamma_{\varepsilon}} \lambda_{j}(t)|d t|=\int_{\hat{\gamma}_{\varepsilon}} \hat{\lambda}(z)|d z| \geqq \int_{\hat{\gamma}_{\delta}} \tau(z)|d z| .
$$


But $\int_{\hat{\gamma}_{\varepsilon}} \tau(z)|d z|$ is not less than the distance from the circle $|z|=1$ to the circle $|z|=\varepsilon^{1 / n}$ in the metric $\tau(z)|d z|$, that is,

$$
\begin{aligned}
\int_{\hat{\gamma}_{\varepsilon}} \tau(z)|d z| & \geqq \int_{\varepsilon^{1 / n}}^{1} \frac{a}{1-a^{2} x^{2}} d x \\
& =\frac{1}{2} \log \left(\frac{1+a}{1+a \varepsilon^{1 / n}} \frac{1-a \varepsilon^{1 / n}}{1-a}\right)>\frac{1}{2} \log \frac{1-a \varepsilon^{1 / n}}{1-a},
\end{aligned}
$$

and therefore

$$
\int_{\gamma_{\varepsilon}} \lambda_{f}(t)|d t|>\frac{1}{2} \log N_{n}
$$

where $N_{n}=\left(1-a \varepsilon^{1 / n}\right) /(1-a), a=\left(\left(4 c^{2} n^{2}+1\right)^{1 / 2}-1\right) / 2 c n$. We have proven this inequality under the assumption that $\nu_{k}^{j}=n<\infty$. We remark now that if $\nu_{k}^{j}=\infty$, then it holds for every $n$. This follows at once from Theorem 4.

Next, for $n \rightarrow \infty$ we have

$$
\begin{aligned}
a & =\left(1+1 / 4 c^{2} n^{2}\right)^{1 / 2}-1 / 2 c n=1-1 / 2 c n+O\left(1 / n^{2}\right), \\
\varepsilon^{1 / n} & =1+(\log \varepsilon) / n+O\left(1 / n^{2}\right),
\end{aligned}
$$

so that

$$
N_{n}=\left(1-a \varepsilon^{1 / n}\right) /(1-a)=1+2 c \log (1 / \varepsilon)+O(1 / n)
$$

Thus by (3),

$$
\liminf _{j \rightarrow \infty} \int_{y_{\varepsilon}} \lambda_{j}(t)|d t|>\log (1+2 c \log (1 / \varepsilon))^{1 / 2}
$$

Hence

$$
\int_{\gamma_{\varepsilon}} \sigma(t)|d t|>\log (1+2 c \log (1 / \varepsilon))^{1 / 2}
$$

and

$$
\int_{\gamma} \sigma(t)|d t|=\lim _{\varepsilon \rightarrow 0} \int_{\gamma_{\varepsilon}} \sigma(t)|d t|=\infty
$$

as asserted.

By Theorem 2, we conclude that $\sigma(t)=\lambda(t)$ on $S$. The proof of Theorem 5 is complete.

VII. The limit theorem for Fuchsian groups. As above, let $S, \boldsymbol{P}, \boldsymbol{\nu}, \boldsymbol{\nu}^{j}, j=1,2,3$, $\ldots$, be given such that the Riemann surfaces with signature $(S, P, v),\left(S, P, v^{j}\right)$, $j=1,2,3, \ldots$, are nonexceptional. Then they can be represented by Fuchsian groups $G, G_{j}, j=1,2,3, \ldots$, with natural projections $g, g_{j}, j=1,2,3, \ldots$, from the unit disk onto the respective Riemann surfaces with signature. For the sake of uniformity in notation, we shall write $G_{\infty}, g_{\infty}$ for $G, g$ respectively. We shall 
normalize $g_{\infty}, g_{j}$ in the following manner. Let $P$ be a point fixed in $S_{0}$, and let a direction $v$ at $P$ be fixed. We normalize $g_{\infty}, g_{j}$ by the condition that the origin 0 of the unit disk is mapped onto $P$ and the direction of the positive real axis onto the direction $v$. Then $g_{\infty}, g_{j}, G_{\infty}, G_{j}$ are uniquely determined. Note that $g_{\infty}: \Delta_{\infty}$ $\rightarrow S_{0}, g_{j}: \Delta_{j} \rightarrow S_{0}$, where $\Delta_{\infty}=\Delta-\left\{\right.$ all the preimages of $P_{k}, 1 \leqq \nu_{k}<\infty$, under $\left.g_{\infty}\right\}$, and $\Delta_{j}$ is defined similarly.

Construct the universal covering $\Delta$ of $\Delta_{\infty}$ and normalize the projection $h_{\infty}$ by the conditions that $h_{\infty}(0)=0, h_{\infty}^{\prime}(0)>0$. Also let $f_{\infty}=g_{\infty} \circ h_{\infty}$, then $f_{\infty}$ is the universal covering projection of $S_{0}$ with the property that the origin $0 \in \Delta$ is mapped onto $P$ and the direction of the positive real axis to the direction $v$. Similarly, construct the universal covering $\Delta$ of $\Delta_{j}$, normalize the projection $h_{j}$ by the conditions that $h_{j}(0)=0, h^{\prime}(0)>0$, and let $f_{j}=g_{j} \circ h_{j}$, then $f_{j}$ is the universal covering projection of $S_{0}$ with the property that the origin $0 \in \Delta$ is mapped onto $P$ and the direction of the positive real axis to the direction $v$. It follows that $f_{\infty}=f_{j}$. From now on, we simply write $f$ for $f_{\infty}, f_{j}$.

Let $F$ be the covering group of $f, H_{\infty}$ the covering group of $h_{\infty}, H_{j}$ the covering group of $h_{j}$. It is known that we have the following exact sequences of groups and homomorphisms:

$$
1 \longrightarrow H_{\infty} \leftrightarrow F \stackrel{\chi_{\infty}}{\longrightarrow} G_{\infty} \longrightarrow 1, \quad 1 \longrightarrow H_{j} \leftrightarrow F \stackrel{\chi_{j}}{\longrightarrow} G_{j} \longrightarrow 1,
$$

such that for every $\alpha \in F, h_{\infty} \circ \alpha=\chi_{\infty}(\alpha) \circ h_{\infty}, h_{j} \circ \alpha=\chi_{j}(\alpha) \circ h_{j}$. (See e.g. Bers [4].)

THEOREM 6. Given $S, P, \nu^{0}, \nu, \nu^{j}, j=1,2,3, \ldots$, such that $\nu^{0} \leqq \nu^{j}$ for all $j$ and $\boldsymbol{\nu}^{j} \rightarrow \boldsymbol{\nu}$ as $j \rightarrow \infty$. Assume that $\left(S, \boldsymbol{P}, \boldsymbol{\nu}^{0}\right),(S, \boldsymbol{P}, \boldsymbol{\nu}),\left(S, \boldsymbol{P}, \boldsymbol{\nu}^{j}\right), j=1,2,3, \ldots$, are all nonexceptional Riemann surfaces with signature. Define $h_{j}, g_{j}, h_{\infty}, g_{\infty}, f, H_{j}, G_{j}, H_{\infty}$, $G_{\infty}, F, \chi_{j}, \chi_{\infty}$ as above.

Then $h_{j} \rightarrow h_{\infty}$ and, for every $\alpha \in F, \chi_{j}(\alpha) \rightarrow \chi_{\infty}(\alpha)$ normally on $\Delta$ as $j \rightarrow \infty$.

Proof. (a) We shall first prove that $h_{j}(z) \rightarrow h_{\infty}(z)$ normally on $\Delta$ as $j \rightarrow \infty$. Note that $\left|h_{j}(z)\right|<1, z \in \Delta$, for every $j$. Hence $\left\{h_{j}\right\}$ is a normal family of holomorphic functions. That is, in every infinite sequence of this family, there exists a normally convergent subsequence, say, with limit function $h$. $h$ is holomorphic in $\Delta$. If we can prove that $h=h_{\infty}$, then the original sequence $\left\{h_{j}\right\}$ converges to $h_{\infty}$ normally on $\Delta$ as $j \rightarrow \infty$. We may assume that $h_{j} \rightarrow h$ normally on $\Delta$ as $j \rightarrow \infty$. We shall prove that $h=h_{\infty}$. By assumption, $h_{j}(0)=0, h_{j}^{\prime}(0)>0$. Therefore $h(0)=0, h^{\prime}(0) \geqq 0$. We shall show that in fact $h^{\prime}(0)>0$.

Let $\lambda_{j}(t)|d t|, \lambda_{\infty}(t)|d t|$ be the Poincaré metrics on $S$ defined by $G_{j}, G_{\infty}$. By Theorem 5, $\lambda_{j}(t) \rightarrow \lambda_{\infty}(t)$ on $S_{0}$ as $j \rightarrow \infty$. Lift, by $f$, the metrics $\lambda_{j}(t)|d t|, \lambda_{\infty}(t)|d t|$ on $S_{0}$ to metrics $\rho_{j}(z)|d z|, \rho_{\infty}(z)|d z|$ on $\Delta$. Hence $\rho_{j}(z)|d z|=\lambda_{j}(t)|d t|, \rho_{\infty}(z)|d z|$ $=\lambda_{\infty}(t)|d t|$, with $t=f(z)$, and $\rho_{j}(z) \rightarrow \rho_{\infty}(z)$ on $\Delta$ as $j \rightarrow \infty$. On the other hand, if we lift the metrics $\lambda_{j}(t)|d t|$ on $S_{0}$ to $\Delta_{j}$ by $g_{j}$ and $\lambda_{\infty}(t)|d t|$ on $S_{0}$ to $\Delta_{\infty}$ by $g_{\infty}$, we clearly get back the Poincaré metric on the unit disk. Therefore,

$$
\rho_{j}(z)=\left|h_{j}^{\prime}(z)\right| /\left(1-\left|h_{j}(z)\right|^{2}\right), \quad \rho_{\infty}(z)=\left|h_{\infty}^{\prime}(z)\right| /\left(1-\left|h_{\infty}(z)\right|^{2}\right)
$$


for $z \in \Delta$. Note that $h_{j} \rightarrow h, h_{j}^{\prime} \rightarrow h^{\prime}$ and $\rho_{j} \rightarrow \rho_{\infty}$ as $j \rightarrow \infty$, so that

$$
\rho_{\infty}(z)=\left|h^{\prime}(z)\right| /\left(1-|h(z)|^{2}\right)
$$

for $z \in \Delta$. Since $\rho_{\infty}(0)>0$, it follows that $h^{\prime}(0) \neq 0$, hence $h^{\prime}(0)>0$.

Altogether,

$$
h(0)=0, \quad h^{\prime}(0)>0, \quad \text { and } \quad\left|h^{\prime}(z)\right|=\rho_{\infty}(z)\left(1-|h(z)|^{2}\right), \quad \text { for } z \in \Delta .
$$

We claim that these three conditions determine $h(z)$ uniquely. It suffices to show that they determine all derivatives $h^{(n)}(0), n=1,2, \ldots$ Square $\left(^{*}\right)$ and put $K(z, \bar{z})$ $=\rho_{\infty}^{2}(z)$. We can show that $\left|h^{\prime}(0)\right|^{2}=K(0,0)$. By assumption $h^{\prime}(0)>0$, therefore $h^{\prime}(0)$ is completely determined. Further differentiation gives that $h^{(n)}(0)\left(h^{\prime}(0)\right)^{-}$ $=\partial^{n-1} K(0,0) / \partial z^{n-1}$. Therefore, $h^{(n)}(0)$ is completely determined for all $n$. It follows immediately that $h=h_{\infty}$, as asserted.

(b) We shall prove that for every $\alpha \in F, \chi_{j}(\alpha) \rightarrow \chi_{\infty}(\alpha)$ normally on $\Delta$ as $j \rightarrow \infty$. For simplicity, write $\alpha_{j}$ for $\chi_{j}(\alpha), \alpha_{\infty}$ for $\chi_{\infty}(\alpha)$. Recall that $\alpha_{j} \in G_{j}, \alpha_{\infty} \in G_{\infty}$ are Möbius transformations from $\Delta$ onto itself and $h_{j}(\alpha(z))=\alpha_{j}\left(h_{j}(z)\right), h_{\infty}(\alpha(z))$ $=\alpha_{\infty}\left(h_{\infty}(z)\right)$, for $z \in \Delta$. By part (a), we conclude that $\alpha_{j}\left(h_{j}(z)\right) \rightarrow \alpha_{\infty}\left(h_{\infty}(z)\right), z \in \Delta$, as $j \rightarrow \infty$. From this we shall show that $\alpha_{j} \rightarrow \alpha_{\infty}$ normally on $\Delta$ as $j \rightarrow \infty$.

Now $\left|\alpha_{j}(z)\right|<1$ for all $j$. It follows that $\left\{\alpha_{j}\right\}$ is a normal family of holomorphic functions, that is, any infinite sequence in the family has a normally convergent subsequence. If we can prove that the limit function is always $\alpha_{\infty}$, then $\alpha_{j} \rightarrow \alpha_{\infty}$ normally on $\Delta$ as $j \rightarrow \infty$. As usual, we may assume that $\alpha_{j} \rightarrow \hat{\alpha}$ normally on $\Delta$ as $j \rightarrow \infty$, and we shall prove that $\hat{\alpha}=\alpha_{\infty}$.

Let $z_{i}, i=1,2,3$, be three distinct points in $\Delta$ such that the points $a_{i}=h_{\infty}\left(z_{i}\right)$, $i=1,2,3$, are also distinct. Let $b_{i}=\alpha_{\infty}\left(a_{i}\right)=\alpha_{\infty}\left(h_{\infty}\left(z_{i}\right)\right), i=1,2,3$. Thus $\alpha_{\infty}$ maps $a_{i}$ onto $b_{i}, i=1,2,3$, respectively. On the other hand, let $a_{i}^{j}=h_{j}\left(z_{i}\right), i=1,2,3$. Then by part (a), $a_{i}^{j} \rightarrow a_{i}$ as $j \rightarrow \infty, i=1,2,3$. Let $b_{i}^{j}=\alpha_{j}\left(a_{i}^{j}\right)=\alpha_{,}\left(h_{j}\left(z_{i}\right)\right), i=1,2,3$. From the fact that $\alpha_{j}\left(h_{j}(z)\right) \rightarrow \alpha_{\infty}\left(h_{\infty}(z)\right)$ as $j \rightarrow \infty$, we conclude that $b_{i}^{j} \rightarrow b_{i}$ as $j \rightarrow \infty$, $i=1,2,3$. The fact that $a_{i}, i=1,2,3$, are distinct implies that $a_{i}^{j}, i=1,2,3$, are also distinct for $j$ large. Therefore, for $j$ large, $w=\alpha_{j}(z)$ can be expressed as

$$
\frac{\left(w-b_{1}^{j}\right)\left(b_{2}^{j}-b_{3}^{j}\right)}{\left(w-b_{3}^{j}\right)\left(b_{2}^{j}-b_{1}^{j}\right)}=\frac{\left(z-a_{1}^{j}\right)\left(a_{2}^{j}-a_{3}^{j}\right)}{\left(z-a_{3}^{j}\right)\left(a_{2}^{j}-a_{1}^{j}\right)}
$$

It follows that the limit $\hat{\alpha}$ of $\alpha_{j}, w=\hat{\alpha}(z)$, can be expressed as

$$
\frac{\left(w-b_{1}\right)\left(b_{2}-b_{3}\right)}{\left(w-b_{3}\right)\left(b_{2}-b_{1}\right)}=\frac{\left(z-a_{1}\right)\left(a_{2}-a_{3}\right)}{\left(z-a_{3}\right)\left(a_{2}-a_{1}\right)}
$$

Therefore $\hat{\alpha}$ is a Möbius transformation mapping the distinct points $a_{i}$ onto $b_{i}$, $i=1,2,3$, respectively. It follows that $\hat{\alpha}=\alpha_{\infty}$. 


\section{REFERENCES}

1. L. Ahlfors and L. Sario, Riemann surfaces, Princeton Math. Series, no. 26, Princeton Univ. Press, Princeton, N. J., 1960. MR 22 \#5729.

2. L. Bers, Pseudoanalytic functions, Notes, New York University, New York, 1949.

3. L. Bers, F. John and M. Schechter, Partial differential equations, Proc. Summer Seminar (Boulder, Colorado, 1957), Lectures in Appl. Math., vol. 3, Interscience, New York, 1964. MR 29 \#346.

4. L. Bers, Riemann surfaces, Notes, New York University, New York, 1958.

5. —_, Uniformization by Beltrami equations, Comm. Pure Appl. Math. 14 (1961), 215-228. MR 24 \#A2022.

6. R. Courant and D. Hilbert, Methods of mathematical physics. II: Partial differential equations, Interscience, New York, 1962. MR 25 \#4216.

7. L. Ford, Automorphic functions, 2nd ed., Chelsea, New York, 1951.

8. M. Heins, On Fuchsoid groups that contain parabolic transformations, Contribution to Function Theory, Internat. Colloq. Function Theory (Bombay, 1960), Tata Institute of Fundamental Research, Bombay, 1960, pp. 203-210. MR 27 \#1597.

9. O. Kellogg, Foundations of potential theory, Ungar, New York, 1929.

10. P. Koebe, Ueber die Uniformisierung beliebiger analytischer Kurven, Gött. Nachr. (1907), 191-210.

11. J. Lehner, Discontinuous groups and automorphic functions, Math. Surveys, no. 8, Amer. Math. Soc., Providence, R.I., 1964. MR 29 \#1332.

IBM Thomas J. Watson Research Center, Yorktown Heights, New York 10598 